\title{
Main topic
}

ce part of the patients experience considerable toxicity in prolonged therapy, individualizing slightly the treatment dose and intermittent administration may be better tolerated and still improve the treatment results [3]. The median 19.3 months' survival and a few late objective responses after 12 months of treatment support longterm treatment schedules for stable and responding patients.

\section{Corresponding author Prof. S. Pyrhönen}

Department of Oncology and Radiotherapy, Turku University Central Hospital, Kiinamyllykatu 4-8, SF-20520 Turku, Finland e-mail: seppo.pyrhonen@tyks.fi

Conflict of interest: No information supplied

\section{References}

1. Medical Research Council Renal Cancer Collaborators (1999) Interferon-alpha and survival in metastatic renal carcinoma: early results of a randomized controlled trial. Lancet 353:14-17

2. Pyrhönen S, Salminen E, Ruutu M et al. (1999) Prospective randomized trial of interferon alfa2a plus vinblastine versus vinblastine alone in patients with advanced renal cell cancer. J Clin Oncol 17:2859-2867

3. Kankuri M, Pelliniemi T-T, Pyrhönen S et al. (2001) Feasibility of prolonged use of interferon- $\alpha$ in metastatic kidney carcinoma. Cancer 92:761-767

Urologe [A] 2004 · [Suppl 2]43:S138-S140 · DOI 10.1007/s00120-004-0605-7

Online publiziert: 18. May 2004 - C Springer Medizin Verlag 2004

\author{
U. Keilholz \\ Department of Medicine III, Charité, Campus Benjamin Franklin, Berlin, Germany
}

\section{Immunotherapy in non-renal carcinomas}

\section{Cytokine treatment}

After initial enthusiasm, cytokine treatment has proved valuable only in renal cell cancer, melanoma, nasopharyngeal carcinoma, B-lymphocyte-derived neoplasms, and myeloproliferative disorders. Since the mechanism of action of interferon in hematopoietic neoplasms and nasopharyngeal carcinoma is most likely not immunological, the only non-renal neoplasm where cytokines have clinically relevant activity as immunotherapeutic agents is melanoma. For adjuvant treatment in patients with stage III melanoma, interferon- $\alpha$ is licensed based an several phase III trials demonstrating an impact on progression-free survival, and in two studies also on overall survival. However, there is still considerable controversy regarding the optimal dose and schedule, and also whether the effect of interferon- $\alpha$ is immunologic or whether it acts mainly through anti-angiogenetic mechanisms.

This leaves interleukin-2(IL-2)-based treatment of stage IV melanoma as the only disease, and situation of a non-renal cancer, in which cytokine-based immunotherapy has proven efficacy. In the USA, IL-2 is licensed for stage IV melanoma based on the observation in all larger clinical trials that many patients with metastatic melanoma, who achieve a complete remission after IL-2 treatment, enjoy longterm freedom from recurrence $[1,2]$. In Europe, this license has not been granted. After several randomized trials of chemoimmunotherapy failed to demonstrate any clinically relevant activity of IL-2 in this setting (M.B. Atkins, personal communication) $[3,4,5]$, current research is focused on methods to improve the promising immunological activity of IL-2 by combination with vaccines [6] or with histamine [7].

\section{Vaccines}

Efficient cancer vaccines have been the dream of tumor immunologists for more than a century. The immunologic discoveries described in the introduction have facilitated the rational development of vaccine approaches in the last decade. We now see accumulating evidence from a number of vaccine studies that modern vaccines with specific tumor antigens are able to elicit immune responses, and that clinically meaningful effects can be observed, especially in patients with low-volume or occult disease. The clinical trials can be grouped according to the class of tumor antigen, the class of compound, and the adjuvant used to augment immunogenicity.

\section{Vaccine antigens}

In most vaccine studies, cancer-testis antigens (e.g., the MAGE family) or differentiation antigens (melanosomal antigens, carcinoembryonic antigen, prostate-specific antigen, etc.) have been used. These antigens have in common that they are expressed in some normal cells at a low level and that they are not essential for tumor cell survival. Thus resistance mechanisms on 
Abstract

the tumor-cell level include antigen-loss as well as antigen-presentation defects. Consequently, only a few and usually shortlived responses have been observed in patients with metastatic tumors, whereas evidence emerges that these vaccines may be able to prevent outgrowth of occult disease in a proportion of patients [8].

Tumor antigens currently evolving are proteins that are essential for tumor cell proliferation, such as transcription factors and telomerase. The fundamental difference with cancer-testis and differentiation antigens is that tumor cells cannot proliferate if they shut down expression of crucial transcription factors, leaving presentation defects as the major resistance mechanism at the tumor cell level. Among these antigens, the Wilms' tumor gene product WT1 deserves attention, since this transcription factor is crucial for proliferation in the majority of acute leukemias and a large variety of carcinomas, and clinical responses of surprising durability in leukemia patients have been reported at several recent meetings.

The third approach is to use the complexity of the tumor proteome for vaccination, either based on the patient's own tumor or on a panel of allogeneic tumors.

\section{Vaccine compounds}

For vaccination with specific antigens, peptides, whole proteins, and antigen-encoding DNA have been used. Peptides can easily be synthesized and are loaded on free HLA molecules and antigen-presenting cells after intra-/subcutaneous injection. Proteins have the disadvantage of having to be processed by antigen-presenting cells prior to presentation, a mechanism that is not very efficient in humans. DNA encoding tumor antigens has to enter the antigen- presenting cells and be transcribed, which presents another obstacle; however, once transcribed in the cell at a sufficient level, presentation occurs through the efficient natural pathway.

For vaccination with complex antigen mixtures, several approaches have been followed, including extracts from autologous tumors, tumor cell lysates, modified whole tumor cells, or tumor-cell RNA. Among these, tumor-derived heat-shock protein preparations deserve special con- sideration, since it has been shown in this model that vaccination with this complex mixture induces $\mathrm{T}$-cell immunity to specific antigens, and that induction of immunity is linked to clinical efficacy [9]. The background of heat-shock protein vaccination is that physiologically these intracellular chaperones transport peptides from degraded proteins to HLA molecules, thus heat-shock protein preparations contain a sample of peptides from the entire intracellular proteome.

\section{Vaccine adjuvants}

Since almost all tumor antigens are weakly immunogenic self antigens, the importance of adjuvants to increase immunogenicity is much higher than in prophylactic vaccinations against foreign pathogens. Approaches that have been followed are manifold and include specific cytokines (e.g., GM-CSF), foreign helper proteins (e.g., tetanus toxoid, keyhole lympet hemocyanin), and specific helper proteins, injected together with the antigen. Other approaches include mechanisms to increase antigen uptake into antigen-presenting cells, ex vivo loading of antigens onto dendritic cells, and prime-boost approaches with different vaccine preparations. With the current knowledge, we only have very limited information to compare the different adjuvants. However, modern immunologic monitoring methods (as summarized elsewhere, [10]) have been developed that now allow comparison of the immunologic potency of different vaccines and selection of the most promising ones for further clinical development.

\section{Corresponding author \\ Dr. U. Keilholz}

Department of Medicine III, Charité, Campus Benjamin Franklin, Hindenburgdamm 30, 12200 Berlin, Germany e-mail: ulrich.keilholz@medizin-fu-berlin.de

Conflict of interest: No information supplied

\section{References}

1. Keilholz U, Conradt C, Legha S, Khayat D, Scheibenbogen C, Thatcher N et al. (1998) Results of IL-2based treatment in advanced melanoma: a caserecord based analysis of 631 patients. J Clin Oncol 16:2921-2929
Urologe [A] 2004 · [Suppl 2]43:S138-S140

DOI 10.1007/s00120-004-0605-7

๑) Springer Medizin Verlag 2004

\section{U. Keilholz}

\section{Immunotherapy in non-renal carcinomas}

\section{Abstract}

Two discoveries have been crucial for the development of modern immunotherapy to human neoplasia. The first is the technology to produce recombinant proteins in large quantities, pioneered in the late 1970s, that has introduced recombinant cytokines as well as chimeric and humanized antibodies into the clinic. The second is the recognition of short peptides derived from intracellular proteins, which are carried to the cell surface on HLA molecules and present to T lymphocytes a sample of the intracellular protein pool for inspection, which provides the foundation for modern tumor vaccines. In this short review, the principles of and the results with cytokine treatment and vaccines in human neoplasms other than renal cancer are summarized. 


\section{Main topic}

2. Atkins MB, Lotze MT, Dutcher JP et al. (1999) Highdose recombinant interleukin 2 therapy for patients with metastatic melanoma: analysis of 270 patients treated between 1985 and 1993. J Clin Oncol 17:2105-116

3. Keilholz U, Martus P, Punt CJA et al. (2002) Prognostic factors for survival and factors associated with long-term remission in patients with advanced melanoma receiving cytokine-based treatments: second analysis of a randomized EORTC Melanoma Group trial comparing interferon- $\alpha 2 a$ (IFN $\alpha$ ) and interleukin 2 (IL-2) with or without cisplatin Eur J Cancer 38:1501-11

4. Keilholz U, CJA Punt, M Göre, W Kruit, P Patel, D Lienard et al. (2000) Dacarbazine, cisplatin and IFN- $\alpha$ with or without IL- 2 in advanced melanoma (EORTC trial 18951). Ann Oncol 11 [Suppl 4]:4

5. Hauschild A, Garbe C, Stolz W, Ellwanger U, Seiter S, Dummer R et al. (2001) Dacarbazine and interferon alpha with or without interleukin 2 in metastatic melanoma: a randomized phase III multicentre trial of the Dermatologic Cooperative Oncology Group (DeCOG). Br J Cancer 84:1036-42

6. Rosenberg SA, Yang JC, Schwartzentruber DJ et al. (1998) Immunologic and therapeutic evaluation of a synthetic peptide vaccine for the treatment of patients with metastatic melanoma. Nature Med 4:321-327

7. Agarwala SA, Glaspy J, O'Day SJ et al. (2002) Results from a randomized phase 111 study comparing combined treatment with histamine dihidrochloride plus interleukin- 2 versus interleukin2 alone in patients with metastatic melanoma. J Clin Oncol 20:125-133

8. Scheibenbogen C, Nagorsen D, Seliger Bolz U (2002) Long-term freedom from recurrence in 2 stage IV melanoma patients following vaccination with tyrosinase peptides. Int J Cancer 99:403-408

9. Belli F, Testori A, Rivoltini L et al. (2002) Vaccination of metastatic melanoma patients with autologous tumor-derived heat shock protein gp96-peptide complexes: clinical and immunologic findings. J Clin Oncol 20:4169-4180

10. Keilholz U, Weber J, Finke JH et al. (2002) Immunologic monitoring of cancer vaccine therapy: results of a workshop sponsored by the Society for Biological Therapy. J Immunother 25:97-138

Urologe [A] 2004 · [Suppl 2]43:S140-S144 · DOI 10.1007/s00120-004-0606-6

Online publiziert: 4. June 2004 - @ Springer Medizin Verlag 2004

Edith Huland ${ }^{1} \cdot$ H. Heinzer ${ }^{1}$ R. A. Jörres ${ }^{2} \cdot$ D. Loppow ${ }^{2} \cdot$ H. Huland ${ }^{1}$

${ }^{1}$ Department of Urology, University Hospital Hamburg-Eppendorf, Hamburg, Germany

${ }^{2}$ Hospital Großhansdorf, Großhansdorf, Germany

\section{Therapeutic approaches in metastatic renal cell carcinoma: local immunotherapy}

\section{Introduction}

Interleukin-2 (IL-2) and/or interferon-alpha $(\mathrm{IFN}-\alpha)$ induce impressive remissions and prolong life in patients carefully selected for a possibly toxic treatment. The design of treatment protocols is significantly influenced by the toxicity of systemic cytokine therapy, such as flu-like symptoms, vascular leakage, and fatigue. Patient selection for systemic immunotherapy is determined by the patient's ability to tolerate its side effects rather than their therapeutic need. There is an urgent need for well-tolerated effective therapies, especially for patients with significant comorbidities as well as for those resistant to current systemic cytokine treatment. Median survival in metastatic disease without treatment is about 7 months, with very few long-term survivors [1].

The major toxicity of IL-2 is directly - and nearly exclusively - linked to intravascular ("systemic") IL-2 concentrations [2]. The major antitumor activity of IL-2, however, depends on the activation of immune cells, preferably local immune cells such as tumor-infiltrating lymphocytes. Since 1990, aerosol IL-2 has been used to treat pulmonary metastatic renal cell carcinoma (pmRCC). Aerosol therapy plays a major role in the treatment of various lung diseases. Here we report data concerning safety and efficacy of local aerosol application using IL-2 in pmRCC.
Methods

Study I

Study design and patient population: "exclusive inhalation" The tolerability and efficacy of inhalational natural IL-2 were analyzed in a multicenter, open, nonrandomized clinical phase II study according to Good Clinical Practise in 24 patients with pmRCC treated from August 1992 to December 1994 at nine research centers in Germany.

Patients of all centers had to have prior progression of their pulmonary metastatic disease confirmed by central radiologic assessment. The radiologist was also in charge of response evaluation during therapy.

\section{Inhalation therapy}

Natural IL-2 was given as an undiluted watery solution (concentration 100,000 biologic response modifier program units/ml) in three dose levels (week 0 $12,5 \times 200,000$ units per day; week $13-$ $24,5 \times 300,000$ units per day; week 25 $48,5 \times 400,000$ units per day). Natural IL2 was administered by means of an inhaling apparatus (Salvia Lifetec A 200, Kronberg, Germany) on each study day five times per day and constituted the only anticancer therapy given during the study period. 